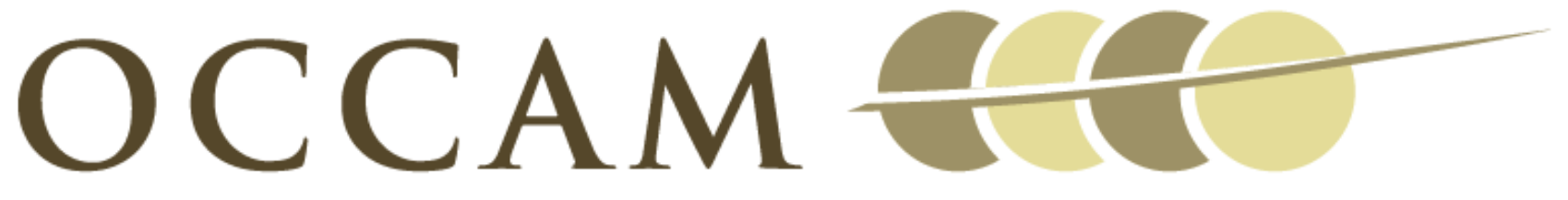

OXFORD CENTRE FOR COLLABORATIVE APPLIED MATHEMATICS

Report Number 11/49

The kinetics of ice-lens growth in porous media by

Robert W. Style and Stephen S. L. Peppin

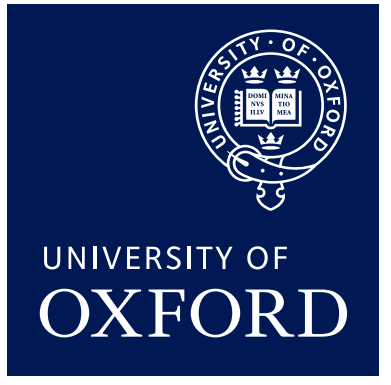

Oxford Centre for Collaborative Applied Mathematics Mathematical Institute 24 - 29 St Giles'

Oxford

OX1 3LB

England 



\title{
The kinetics of ice-lens growth in porous media
}

\author{
ROBERT W. STYLE't, AND STEPHEN S. L. PEPPIN \\ ${ }^{1}$ Oxford Centre for Collaborative Applied Mathematics, University of Oxford, Mathematical \\ Institute, 24-29 St. Giles', Oxford, OX1 3LB, UK
}

(Received ?; revised ?; accepted ?. - To be entered by editorial office)

We analyse the growth rate of segregated ice (ice lenses) in freezing porous media. For typical colloidal materials such as soils we show that the commonly-employed Clapeyron equation is not valid macroscopically at the interface between the ice lens and the surrounding porous medium owing to the viscous dynamics of flow in premelted films. This gives rise to an 'interfacial resistance' to flow towards the growing ice which causes a significant drop in predicted ice-growth (heave) rates and explains why many previous models predict ice-growth rates that are much larger than those seen in experiments. We derive an explicit formula for the ice-growth rate in a given porous medium, and show that this only depends on temperature and on the external pressures imposed on the freezing system. This growth-rate formula contains a material-specific function which can be calculated (with a knowledge of the of the geometry and material of the porous medium), but which is also readily experimentally-measurable. We apply the formula to plate-like particles, and obtain good agreement with previous experimental data. Finally we show how the interfacial resistance explains the observation that the maximum heave rate in soils occurs in medium-grained particles such as silts, while heave rates are smaller for fine- and coarse- grained particles.

\section{Key words:}

\section{Introduction}

The growth of ice in porous media and particle suspensions is an important phenomenon that is a key part of many different processes, examples being the freezing of soils (Dash et al. 2006), colloidal suspensions (Deville et al. 2007), foods (Roos 1995) and biological materials (Benson 2008). In each of these situations the ice growth plays an important role, either in determining the structure of the frozen material (e.g. the control of ice-crystal size in ice-cream manufacture), or because of its ability to cause damage to the porous medium (e.g. cell rupture during cryopreservation). However despite much theoretical work on the growth of ice in porous media, there still remain many questions about the process.

One of the main reasons that ice can be so disruptive to the structure of a freezing porous media, is that ice does not merely solidify the water in the pores of the material. Ice can also push open a pore, growing by sucking water towards it through the surrounding material, which leads to the growth of macroscopic volumes of segregated ice, or ice lenses (e.g. Vlahou \& Worster 2010). This segregated ice is directly responsible for material damage in cells (Benson 2008), and frost heave in soils (Rempel et al. 2004). In 
colloidal suspensions, the process of ice segregation has been recognised as a useful technique for moulding the colloidal constituents into lightweight materials with many useful engineering applications (Deville et al. 2007). Given the importance of ice segregation, we particularly need to understand what controls the growth rate of a segregated ice lens. However it has proven difficult to predict this rate without the use of empirical laws, as experimental growth rates are typically significantly slower than theoretical predictions (we expand on this later). In this paper we shall focus on this problem of determining ice-growth rates. We show that in many typical freezing situations, it is not the hydraulic resistance of the porous medium itself that controls the flow of water towards a growing ice lens (and hence the growth rate of the ice). Instead, the ice-growth rate is controlled by the dynamics of water flow in the interfacial region at the ice-lens/porous-medium boundary. We provide quantitative estimates of this effect, and show that the results can be used to explain previous experimental work. Furthermore we demonstrate how, by taking account of the interfacial effects, we can explain the experimental observation that ice-lens growth rates in fine- and coarse-grained materials are low, while maximum growth rates occurs in materials with intermediately-sized grains.

In analysing the physics of ice growth in porous media, we shall consider the simple, one-dimensional model shown in figure 1(a). A porous medium (or compacted particulate suspension) is frozen from the top down. An ice lens is assumed to form at the surface, and thermomolecular pressure gradients cause water to be sucked up towards the lens through the porous medium, a process commonly referred to as cryosuction. At the base of the system, there is assumed to be a source of water that is maintained at constant pressure. We shall particularly relate this system to the growth of ice lenses in freezing soils, as there is a large body of previous work in this field. Then the reservoir corresponds to a source of groundwater, known to be a necessary prerequisite for the growth of ice lenses, and thus frost heave (Rempel et al. 2004). However the system can also be related to many other configurations. For instance, in the freezing of cells, the porous medium corresponds to a cell membrane with ice on one side and liquid on the other. Similar configurations are also seen in the freezing of colloidal suspensions (e.g. Peppin et al. 2006). Thus it will be seen that our theory can be readily applied to many freezing situations simply by modifying the appropriate system parameters.

As already mentioned, the key step in the growth of a segregated ice lens is the suction of water towards a growing body of ice in a porous material, however the source of this pressure reduction at the ice front may seem rather unintuitive. Its source can be elucidated by considering the ice/porous-medium interface on a microscopic scale, as shown in figure $1(\mathrm{~b})$. Where the ice lens meets the porous material there are repulsive, intermolecular forces between the constituent material of the porous medium and the ice. These forces manifest themselves as a 'disjoining pressure' $p_{T}$ that pushes apart the ice and the porous material, leaving an intervening, microscopically-thin layer of water (a 'premelted film') of thickness $d$. These films wet the porous material/ice surface despite the fact that the temperature is below the melting point of ice, $T_{m}$ (Dash et al. 2006). In order to balance the repulsive forces acting to separate the ice and the particles, the pressure in the liquid films is reduced, and it is this pressure reduction that sucks liquid up towards the growing ice lens (e.g. Rempel et al. 2004).

The thickness of the premelted films is determined from the expression

$$
p_{T}(d)+\gamma_{s l} \mathcal{K}=\rho L_{m} \frac{T_{m}-T}{T_{m}}
$$

(e.g. Dash et al. 2006). Here $\gamma_{s l}$ is the surface tension at an ice-water interface and $\mathcal{K}$ is the curvature of that interface which is defined to be positive when the interface is 

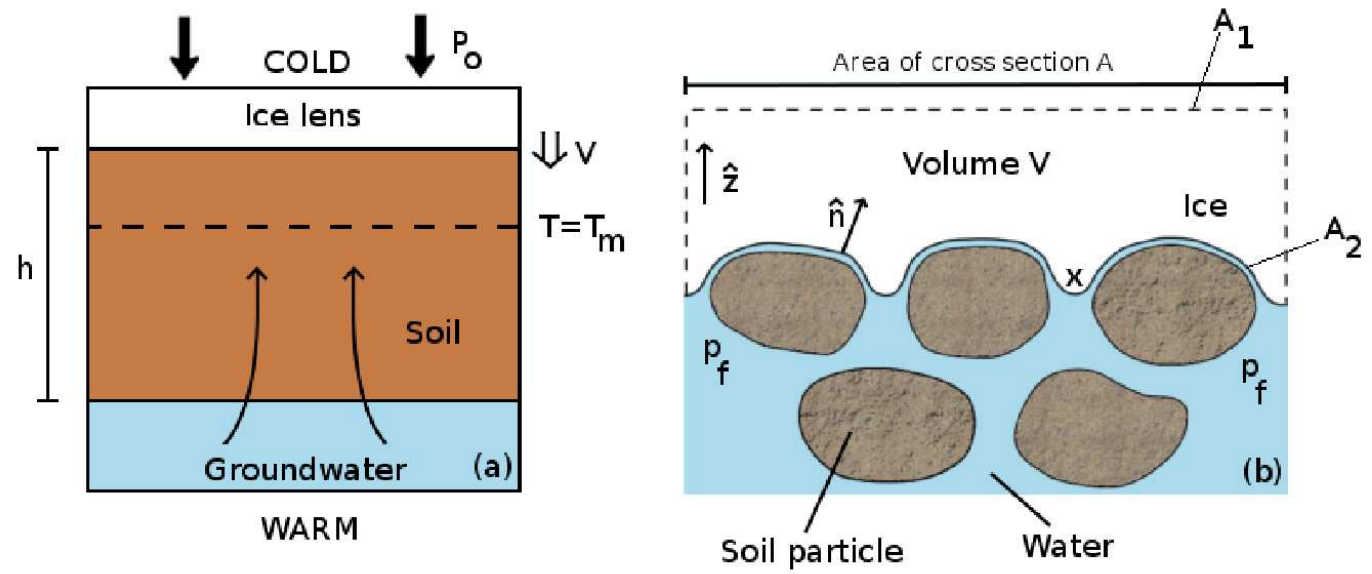

FIGURE 1. Schematic diagram showing ice lenses forming in a freezing porous material. (a) Macroscopic view. Water is sucked up from the groundwater towards the growing ice lens where it freezes. The added mass of water leads to the ice lens expanding at a rate $V$, also causing the material to heave upwards at rate $V$. (b) Microscopic view of particles at the ice-lens/soil interface. The picture is not to scale as the thicknesses of premelted films between the ice and particles are much smaller than the particle sizes. All variables shown in the diagrams are defined in the text.

convex out of the solid (such as at the point marked $\mathrm{x}$ in figure $1(\mathrm{~b})$ ). $\rho$ is the density of ice, assumed to be equal to that of water, $L_{m}$ is the latent heat of melting of ice, and $T$ is the local temperature. The functional dependence of $p_{T}$ on $d$ depends upon the nature of the repulsive intermolecular forces between the ice and porous medium, and these are reviewed by Dash et al. (2006). Typically values for $d$ are in the range $0.2 \mathrm{~nm}-50 \mathrm{~nm}$ (Roseberg 2005). In addition to determining the premelted film thickness, this equation also demonstrates why ice does not grow from the ice lens into the pores of the compacted porous medium as soon as $T<T_{m}$, as shown schematically in figure 1(b). Consider the equilibrium between the ice and pore water at the point ' $x$ ' in figure 1 (b). In this region, away from the thin, premelted films that separate the ice and the porous material, the disjoining pressure is negligible as it only acts on distances that are much smaller than typical pore sizes (e.g. Worster \& Wettlaufer 1999). Thus equation (1.1) becomes $\gamma_{s l} \mathcal{K}=\rho L_{m}\left(T_{m}-T\right) / T_{m}$, the Gibbs-Thomson equation. This equation describes the fact that the freezing point of a solid surface is reduced when the surface has positive curvature. Ice growing into a pore takes the form of a curved meniscus of constant curvature, much like an air bubble does as it grows into a saturated pore. As the ice grows further into a pore, the curvature of the interface increases until it reaches an equilibrium configuration where it satisfies the Gibbs-Thomson condition (such as is shown schematically in figure 1(b)). Ice can only break into the pores of the material when the equilibrium curvature $\mathcal{K}$ becomes smaller than the radius of the pore throats $R_{p}$. At this point, using the Gibbs-Thomson equation, we find that the temperature is given by

$$
T_{i e}=T_{m}-\frac{T_{m} \gamma_{s l}}{\rho L_{m} R_{p}}
$$

the ice-entry temperature (e.g. Peppin et al. 2006).

There are two distinct regimes in which segregated-ice growth can occur. Firstly, when $T_{m} \geqslant T \geqslant T_{i e}$ ice lenses grow before ice penetrates into the pores of the porous material. 
In this case, water is easily transported towards the freezing front and the lens growth is expected to be relatively fast. In the frost-heave literature, early models refer to this as 'primary heave', and assume that only a single ice lens forms at the soil surface rather than multiple ice lenses in the interior of the material (Miller 1977). This assumption was 'necessary' because no known mechanism existed to explain the experimentallyobserved formation of multiple lenses in this regime before ice entry into the soil pores (e.g. Beskow 1935; Miller 1977). Recently Style et al. (????) have shown that multiple ice lenses can indeed form in this regime via a fracture mechanism in the soil whereby new lenses nucleate due to tensile stresses that arise because of cryosuction of water towards growing ice lenses. When the temperature at the ice-lens/porous medium interface drops below $T_{i e}$, ice enters the pores forming a frozen fringe (Rempel et al. 2004; Miller 1977). In this case, blocking of pores by ice hinders transport of the liquid towards growing ice lenses and the ice-lens growth rate is expected to be slower (e.g. the results of Rempel et al. (2004) show that, for a typical soil, the heave rate drops off soon after ice enters the soil pores). Because ice-lens growth should be relatively fast without a frozen fringe, and thus can be considered the more dangerous mode of ice growth, we only consider here ice-lens growth before ice-entry occurs (see Style et al. (????) for a discussion of how ice lensing occurs without the presence of a frozen fringe in typical soils).

In order to determine the suction at the ice-lens/porous-medium interface that brings liquid towards the freezing front, we also need an expression for local thermodynamic equilibrium between water and ice at the interface. This is given by the Clapeyron equation

$$
P=p+\rho L_{m} \frac{T_{m}-T}{T_{m}},
$$

where $p$ is the pressure in the liquid, and $P$ is the pressure in the adjacent solid. This equation demonstrates directly that as the temperature reduces, $p$ must reduce, and it is this suction that draws liquid up towards ice lenses in the porous material. In fact this equation is used in deriving equation (1.1) which determines the thickness of premelted films (e.g. Dash et al. 2006). It is important to note that the equation is valid locally, and is not necessarily valid on a macroscopic scale. For instance, the equation relates the local liquid pressure in the thin films to the local solid pressure in the overlying ice (on the microscopic scale shown in figure 1b). However, a key result that we show in this paper is that this equation does not generally hold when relating the macroscopic pressure of ice in the ice lens to the macroscopic (Darcy) liquid pressure in the pore liquid adjacent to the ice $p_{f}$. Thus if there is an overburden pressure $P_{o}$ pushing down on the top of the soil so that the macroscopic ice pressure in a lens is $P_{o}$, we cannot assume that the Darcy pressure of the liquid in the porous medium adjacent to the lens is equal to the macroscopic equilibrium value $p_{c l}(T)$ (that is a function of the local temperature at the ice/porous-medium interface), that from equation (1.3) is given by

$$
p_{c l}=P_{o}-\rho L_{m}\left(T_{m}-T\right) / T_{m} .
$$

The assumption that $p_{f}=p_{c l}(T)$ is commonly made, for instance having been suggested by Penner \& Goodrich (1981) and used by Konrad (1987), while the model of Rempel et al. (2004) reduces to this assumption when there is no pore ice present. In this paper we shall provide a more general relationship that reduces to equilibrium assumption at very low ice-growth rates.

An indication that the assumption that $p_{f}=p_{c l}(T)$ is not appropriate in typical frost heaving situations can be found by calculating expected ice-growth rates and comparing these with frost-heave experiments. Assuming that the porous medium is incompressible, we can use Darcy's law to determine the flow rate of liquid towards the lens (and thus 


$\begin{array}{lcc}\text { Constant } & \text { Value } & \text { Units } \\ h & 0.05 & \mathrm{~m} \\ \phi & 0.64 & - \\ k_{-} & 10^{-16} & \mathrm{~m}^{2} \\ k_{o} & 2 \times 10^{-15} & \mathrm{~m}^{2} \\ L_{m} & 3.3 \times 10^{5} & \mathrm{~J} \mathrm{~kg}^{-1} \\ \mu & 1.7 \times 10^{-3} & \mathrm{~kg} \mathrm{~m}^{-1} \mathrm{~s}^{-1} \\ P_{o} & 10^{5} & \mathrm{~Pa} \\ p_{R} & 10^{5} & \mathrm{~Pa} \\ \rho & 10^{3} & \mathrm{~kg} \mathrm{~m}^{-3} \\ R_{o} & 1.1 \times 10^{-6} & \mathrm{~m} \\ T_{m} & 273 & \mathrm{~K}\end{array}$

TABLE 1. Data specific to experiments on a soil consisting of spherical silica particles (Watanabe 2002; Watanabe et al. 2001).

the lens growth rate). For instance, if there is a supply of groundwater at a pressure $p_{R}$ at a distance $h$ from the lens (as shown in figure 1), then the flow rate is given by

$$
V=-\frac{k}{\mu} \frac{d p}{d z}=\frac{k}{\mu} \frac{p_{R}-p_{c l}}{h},
$$

where $k$ is the saturated permeability of the soil, $\mu$ is the dynamic viscosity of the liquid, and we have used the assumption that $p_{f}=p_{c l}(T)$. We can compare this expression to ice-growth rates observed in experiments such as those performed by Watanabe (2002). Parameters relating to his experimental system are given in table 1 . Using these parameters with equations (1.4) and (1.5), we find that $V=2.8 \times 10^{-5}\left(T_{m}-T\right) \mathrm{m} \mathrm{s}^{-1}$. However Watanabe found experimentally that the actual ice-growth rate was $V \approx$ $5 \times 10^{-7}\left(T_{m}-T\right) \mathrm{ms}^{-1}$, almost two orders of magnitude slower. We have seen similar results in our own experiments on the freezing of kaolinite. Usually this discrepancy is ascribed to the growth of ice into the pores of the porous material, which blocks the pores, significantly reducing the permeability of the soil and hence the flow rate (e.g. Miller 1977). However Watanabe was able to use Raman spectroscopy to scan for the presence of pore ice, and found no evidence of any ice ahead of (warmer than) the icelens base. This rules out pore blocking by ice, implying that there must be an alternative explanation for the unexpectedly slow ice-growth rates. We suggest that the discrepancy is caused by the incorrect use of the Clapeyron equation to determine the liquid pressure at the ice-lens front. In fact this has previously been suggested, both theoretically and experimentally. For instance Worster \& Wettlaufer (1999) calculated the ice-growth rates in a soil by considering the microscopic flow of liquid around a soil consisting of spherical particles. They showed that there is a large resistance to flow caused by pressure drops in the premelted films between ice and particles. This added effect leads to a significant fall in the ice-growth rate and implies that the assumption that $p_{f}=p_{c l}(T)$ does not hold. Ozawa \& Kinosita (1989) measured the growth of ice on a microporous filter and found that the growth rate of the ice was not controlled by the resistance to flow in the filter, but by the rate at which water could move along the premelted films at the ice-lens front.

In this paper we extend the work of Worster \& Wettlaufer (1999) to consider the growth of ice lenses in general soils and porous media. We find that $p_{f}$ satisfies a kinetic condition $p_{f}-p_{c l}=V f\left(T_{m}-T\right)$, where $f$ is a porous-medium-specific function that can be calculated with a knowledge of the particle geometry or measured experimentally. 
This can be used to derive an expression for ice-growth rate that depends only on the temperature and on the pressure difference $P_{o}-p_{R}$, as observed experimentally. We apply our theory to particles with a plate-like geometry. The results show that this kinetic effect is important in typical frost-heaving situations, while also explaining several qualitative features seen in experiments.

\section{Model}

As described above, we need to calculate the Darcy pressure $p_{f}$ in the liquid directly adjacent to the ice lens. In order to calculate this pressure, we first allow for mechanical equilibrium in the system by adapting a technique used by Rempel et al. (2004) to ensure that the soil supports the weight of the overburden pressure $P_{o}$. Consider an area of ice lens $A_{1}$, perpendicular to the growing ice lens, of cross section $A$, and far enough above the ice/porous-medium interface that the ice pressure $P \rightarrow P_{o}$, as shown in figure $1 \mathrm{~b}$. The downwards force due to the overburden on $A_{1}$ is then given by $A P_{o}$. If we consider the force balance on the volume of ice $V$, between $A_{1}$ and the ice-lens/porous-medium interface, then this weight must be matched by an upwards force on the underlying area $A_{2}$ of the interface. The pressure in the ice at $A_{2}$ is given by the Clapeyron equation (1.3), and so the total upwards force on the base of the lens is given by

$$
\hat{\boldsymbol{z}} \cdot \int_{A_{2}} P \hat{\boldsymbol{n}} d A=\hat{\boldsymbol{z}} \cdot \int_{A_{2}}\left(\left(p-p_{f}\right)+p_{f}+\rho L_{m} \frac{T_{m}-T}{T_{m}}\right) \hat{\boldsymbol{n}} d A=A P_{o} .
$$

Here $\hat{\boldsymbol{z}}$ is the unit vector in the $z$ direction, $\hat{\boldsymbol{n}}$ is the unit vertical normal to the ice/water interface (pointing into the ice). This equation represents the dominant force balance on the volume $V$. We note that there may also be shear forces acting on the sides of the volume $V$, however if we assume that $A_{2}$ encompasses an area consisting of many pores, then the contribution of these shear forces will vanish in comparison to the pressure forces calculated above. From the use of the divergence theorem on the volume $V, A \hat{\boldsymbol{z}}=$ $\int_{A_{1}} \hat{z} d A=\int_{A_{2}} \hat{\boldsymbol{n}} d A$. With this result, and assuming that the temperature $T$ does not vary significantly over the ice/water interface, the force balance above becomes

$$
P_{o}-\rho L_{m} \frac{T_{m}-T}{T_{m}}-p_{f} \equiv p_{c l}-p_{f}=\hat{\boldsymbol{z}} \cdot \frac{1}{A} \int_{A_{i l}}\left(p-p_{f}\right) \hat{\boldsymbol{n}} d A .
$$

We note that this result can also be derived by considering the force balance on the soild constituents of the porous medium at the ice-lens front, similarly to previous calculations regarding the movement of particles at freezing interfaces (e.g. Worster \& Wettlaufer 1999; Rempel et al. 2001), however we use the alternative approach here as the derivation is more straightforward.

In order to calculate the value of the right-hand side of equation (2.2) we assume that $p \rightarrow p_{f}$ in the pore fluid outside of the regions where the intermolecular forces are important (e.g. Worster \& Wettlaufer 1999). Thus the only contribution to the integral comes from the premelted films that separate the solid material of the porous medium and the ice. In these films, the film-thickness $d$ is much smaller than their length-scale, which is on the order of the pore radius $R$ and is typically $O(1 \mu \mathrm{m})$. This means that the flow of liquid in the films satisfies the conditions for lubrication theory to be used to calculate the liquid pressure (e.g. Batchelor 1967). We note that despite the relative thinness of the premelted films in comparison to the dimensions of a water molecule, the use of lubrication theory has been seen to be very successful in modelling phenomena in premelted films (e.g. Wettlaufer et al. 1996), thus it is expected to be a good approximation here. There is also some evidence that parameters of bulk water such as viscosity 
can be used to describe water in premelted films (c.f. the discussion by Style \& Worster $(2005))$, however experimental results for flow in nanofilms differ widely on this subject. We shall discuss our choice of parameters later in more detail.

At a given temperature, we assume that on the microscale ice and water at the ice/porous-medium interface will be in local thermodynamic equilibrium. Thus surface curvature and film thickness are given by equation (1.1). This means that the ice interface will adopt an equilibrium geometry that only depends on the temperature, and not on the ice-growth rate $V$ (this assumption was shown to be good for a typical premelted system by Wettlaufer et al. (1996)). If we consider the thin film between the material of the porous medium and the overlying ice, then the pressure in the film $p$ is given by lubrication theory with $p \rightarrow p_{f}$ at the pore mouthes where the film thickness diverges into the 'bulk' pore fluid. Assuming a constant rate of ice growth, at each point in the film liquid freezes onto the ice lens at a constant rate $V$. Then because of the linearity of lubrication theory, $p-p_{f}=V g(\boldsymbol{x}, T)$, where $g(\boldsymbol{x}, T)$ is a function that depends upon the temperature and particle geometry but is independent of $V$. We shall give a specific example later, however this general formula can give us insight into the boundary condition at a growing ice lens, for if we define

$$
f\left(T_{m}-T\right) \equiv-\hat{\boldsymbol{z}} \cdot \frac{1}{A} \int_{A_{2}} g(\boldsymbol{x}, T) \hat{\boldsymbol{n}} d A,
$$

then equation (2.2) becomes

$$
p_{c l}-p_{f}=-\operatorname{Vf}\left(T_{m}-T\right) .
$$

This is a kinetic condition for the pore pressure at the ice/soil interface. $p_{c l}$ is the liquid pressure that is obtained from the Clapeyron equation (1.3). It can be seen that this differs from the actual pore pressure at the ice/soil interface $p_{f}$ by an amount proportional to the growth rate of the ice. Thus for very slow growth speeds, we recover the Clapeyron relationship, as observed experimentally (e.g. Biermans et al. 1978).

We also can use this kinetic boundary condition to calculate the heave rate. Using Darcy's law (1.5), $V=k\left(p_{R}-p_{f}\right) /(\mu h)$, and then by using equation (2.4) we find that

$$
V=\frac{p_{R}-p_{c l}}{\frac{\mu h}{k}+f\left(T_{m}-T\right)} .
$$

The numerator is the pressure difference between the groundwater, and the equilibrium pressure at the ice interface. The denominator consists of two terms; the first part represents the hydraulic resistance to the flow of the porous medium, while the second term represents the resistance due to flow in the thin premelted films around the particles. These two resistances compete to control the flow rate to the growing ice lens. Defining

$$
\beta=\frac{k f\left(T_{m}-T\right)}{\mu h},
$$

then when $\beta \ll 1$ the hydraulic resistance of the porous medium dominates, while when $\beta \gg 1$ the interfacial resistance dominates. We shall see later that $\beta$ is large for typical soils.

It is worth remarking on several points about this result. Firstly, for a given material $f\left(T_{m}-T\right)$ is experimentally measurable, for instance by taking a system and letting $h \rightarrow 0$ (so that $\beta \gg 1$ ). Then $f\left(T_{m}-T\right)=\left(p_{R}-p_{c l}\right) / V$ which is readily measurable. Once this is known, the results should be applicable to predict ice heave rates in any system consisting of the same material (until the point where ice invades the soil pores). Ozawa \& Kinosita (1989) measured flow rates through a micro-porous filter, and found 


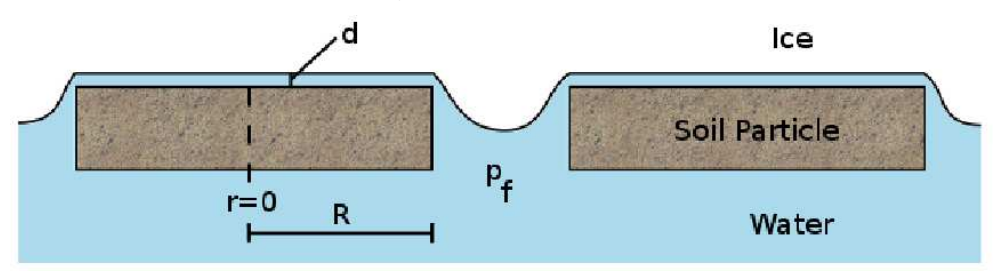

FIGURE 2. Schematic diagram showing the plate-like particle geometry considered in the paper.

that taking $f\left(T_{m}-T\right)$ to be a constant gave good agreement with their observations, while their results show that $\beta \gg 1$.

Secondly, we can use the definition of $p_{c l}$ from equation (1.4) in equation (2.5) to show that $V$ only depends on two parameters, $T_{m}-T$ and the pressure difference $p_{R}-P_{o}$. This simplifies the number of system parameters controlling heave, and so should be useful in the empirical modelling of soil heave. This result also explains the experimental observations of Seto \& Konrad (1994) who looked at the freezing of Devon clayey silt. They varied the overburden pressure $P_{o}$, and 'backpressure' $p_{R}$ together, and found that the heave rates were the same, provided that there was no desaturation of the soil pores.

Thirdly, this equation enables us to calculate the overburden pressure at which there will be no ice-lens growth. Expanding the numerator of equation (2.5), we see that $V=0$ when $P_{o}=p_{R}+\rho L_{m}\left(T_{m}-T\right) / T_{m}$, which is the same result that arises when the kinetic term is not taken into account (Biermans et al. 1978).

\section{Discussion}

\subsection{Application to platelet particles}

In order to demonstrate the use of equation (2.5) for a typical porous medium, we now calculate $f\left(T_{m}-T\right)$ for a particular example of a particulate soil. We consider plate-like particles that make up frost-susceptible clays such as kaolinite and illite. The particles are assumed to be cylindrical, with a circular cross section of radius $R$, and a thickness that is much smaller than $R$. They are expected to orient themselves parallel to the growing ice lens (as shown in figure 2(a)) for two reasons. Firstly, if an ice lens encounters a particle that is orientated at an angle to the freezing front, it will reject the particle ahead of it. Initially this rejective force will only act on the uppermost (coldest) end of the platelet, and this will act to twist the particle around parallel to the growing ice, thus aligning it with the ice lens. Secondly, Perdigon-Aller et al. (2005) conducted experiments on the filtration of kaolinite clay in which the clay was placed on a filter membrane, and water was sucked out of the clay through the membrane. It was observed that the kaolinite platelets had a strong preference to align parallel to the membrane. In the freezing case water is sucked out of the clay at a growing ice lens, so it is effectively equivalent to the membrane-filtration case, but with the ice lens taking the place of the membrane. This suggests that it is likely that platelet particles will lie parallel to a growing ice lens.

For each platelet, the ice/water interface will then take the shape shown in figure 2(a), with a premelted film on the upper side of the particle of constant thickness $d$, and bulk water for $r>R$ (where $r$ is the radial coordinate perpendicular to $\hat{\boldsymbol{z}}$ ), so that $p-p_{f}=0$ when $r=R$. Lubrication theory gives us that the volumetric flow rate in the premelted film, $q$ (per unit length transverse to the flow) is given by

$$
q=-\frac{d^{3}}{12 \mu_{p}} \frac{\partial p}{\partial r}
$$


while mass conservation implies that $2 \pi r q=-\pi r^{2} V$. Here $\mu_{p}$ is the dynamic viscosity of water in the premelted films. By combining these equations and integrating, we then find that

$$
p-p_{f}=\frac{3 \mu_{p} V}{d^{3}}\left(r^{2}-R^{2}\right) .
$$

Thus the total force per unit area of the ice lens surface (i.e. the value of the integral in equation $(2.2))$ is

$$
\frac{\phi}{\pi R^{2}} \int_{0}^{R} 2 \pi r\left(p-p_{f}\right) d r=-\frac{3 \phi \mu_{p} V R^{2}}{2 d^{3}}=-V f\left(T_{m}-T\right)
$$

Here $\phi$ is the area of the ice-lens interface covered by particles, so that $\phi /\left(\pi R^{2}\right)$ is the area of ice lens per particle. From equations (2.5) and (1.4) the ice-growth rate is

$$
V=\frac{p_{R}-P_{o}+\rho L_{m} \frac{T_{m}-T}{T_{m}}}{\frac{\mu h}{k}+\frac{3 \phi \mu_{p} R^{2}}{2 d^{3}}}
$$

while

$$
\beta=\frac{3 k \phi \mu_{p} R^{2}}{\mu h d^{3}}
$$

We can use this result to show that the interfacial resistance is often the rate-controlling process in typical soils. We estimate the permeability $k$ using the Carman-Kozeny equation

$$
k=\frac{R^{2}(1-\phi)^{3}}{100 \phi^{2}},
$$

and assuming that $\mu_{p}=\mu$, we plot the $\beta$ for a range of typical particle sizes, $R$ and film thicknesses $d$ (e.g. Roseberg 2005). This is shown in figure 3. Typical soils have particles of radius greater than $1 \mu \mathrm{m}$ and it can be seen that for such soils, $\beta>1$ for all but the largest film thicknesses (which will occur at temperatures close to $T_{m}$ ). Thus the interfacial resistance overcomes the hydraulic resistance and as such is an important factor that needs to be taken into account.

In fact the calculation shown in figure 3 represents a lower bound for the actual value of $\beta$. This is for two reasons. Firstly, we have assumed that the film viscosity $\mu_{p}$ is equal to the bulk viscosity of water $\mu$. There is some evidence that suggests that this is a reasonable assumption (see the discussion by Style \& Worster 2005), however recent experimental results suggest that, depending on the constituent material of the porous medium, $\mu_{p}$ can be as much as several orders of magnitude larger than $\mu$ (Pittenger et al. 2001; Butt et al. 2000; Goertz et al. 2007). $\beta$ depends linearly upon $\mu_{p}$, so any increase in film viscosity will lead to an increased importance of the interfacial resistance. Secondly, we have assumed that the particle surface is perfectly flat. If there is any surface roughness of the soil particles, there will be an increased resistance to flow through the films (e.g. Gao et al. 2000). This can be thought of as a reduction in the film thickness $d$ to an effective film thickness $d_{e f}<d$ seen by the flow. From equation (3.5), we see that this will again lead to an increase in $\beta$.

\subsection{Comparison with experiment}

In order to compare the equation for the ice-growth velocity (3.4) with experimental data, we need experimental data for the film thickness $d$ as a function of temperature. We are currently unaware of any experimental measurements of $d$ for clays or silts, and so we cannot compare the theory with measurements of ice growth in clays. However Watanabe (2002) measured ice-growth rates for ice in a soil consisting of silica microspheres (of 


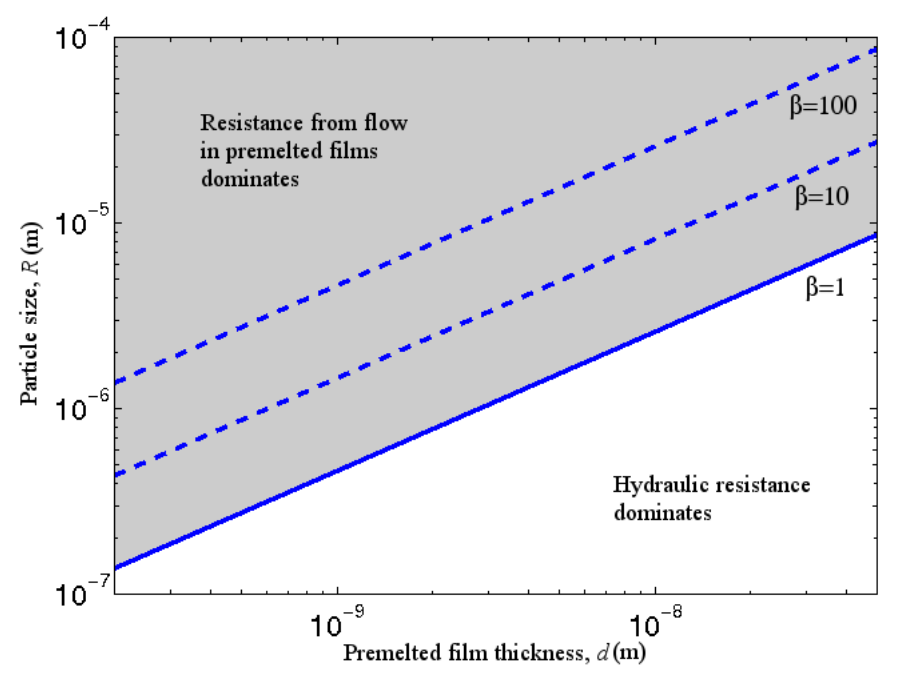

FIGURE 3. Plot showing $\beta$ as a function of particle radius $R$, and the premelted film thickness $d$ for typical values of $R$ and $d$. The grey area shows the regime where $\beta>1$, i.e. the regime where the interfacial resistance dominates the hydraulic resistance.

average radius $R=1.1 \mu \mathrm{m}$, and $R$ being approximately in the range $0.8-1.5 \mu \mathrm{m}$, with the range being estimated from Watanabe \& Mizoguchi 2000), and there is experimental data available for premelted-film thicknesses at a silica/ice interface. Ideally, in order to make a proper comparison, we should also calculate $f\left(T_{m}-T\right)$ for spherical particles. At small undercoolings Worster \& Wettlaufer (1999) have calculated a similar integral to that given in equation (2.2) (by using geometric simplifications), and their results can be used to calculate $f\left(T_{m}-T\right)$. However their geometric assumptions mean that their results only hold while $T_{m}-T \ll 0.05 \mathrm{~K}$. At larger undercoolings the integral cannot be solved analytically due to the significant complexities of the spherical geometry. In order to compare our results with experiments at larger undercoolings, we assume that ice growth in a porous medium consisting of spherical particles behaves similarly to one consisting of platelet particles. This is expected to be a reasonable first approximation.

Engemann et al. (2004) have measured the thickness of premelted films on a silica substrate, and found that

$$
d=a_{0} \ln \left(\frac{17}{T_{m}-T}\right)
$$

where they give $a_{0}=0.84 \mathrm{~nm}$. Using this expression in equation (3.4) we can plot the predicted ice-growth rate as a function of undercooling for Watanabe's experiments (Watanabe 2002). In particular, we use values of $P_{o}, p_{R}, R_{o}$ and $k_{0}$ taken from table 1 . The results are plotted in figure 4 . The figure shows three different curves for predicted ice-growth rate: firstly the hydrodynamic model, assumed by authors such as Rempel et al. (2004), Penner \& Goodrich (1981) and Konrad (1987), where the interfacial viscous effects are absent (dashed curve), secondly the case where we include interfacial effects and using bulk parameters for the premelted films (continuous curve), and thirdly the case where we include interfacial effects and using a fitting parameter for the liquid characteristics in the premelted films (dash-dotted curve) in order to match experimental data from from Watanabe (2002) (crosses). The fitting parameter was selected by setting $\mu_{p}=41 \mu$, which would suggest that the viscosity in the premelted films is significantly larger than 


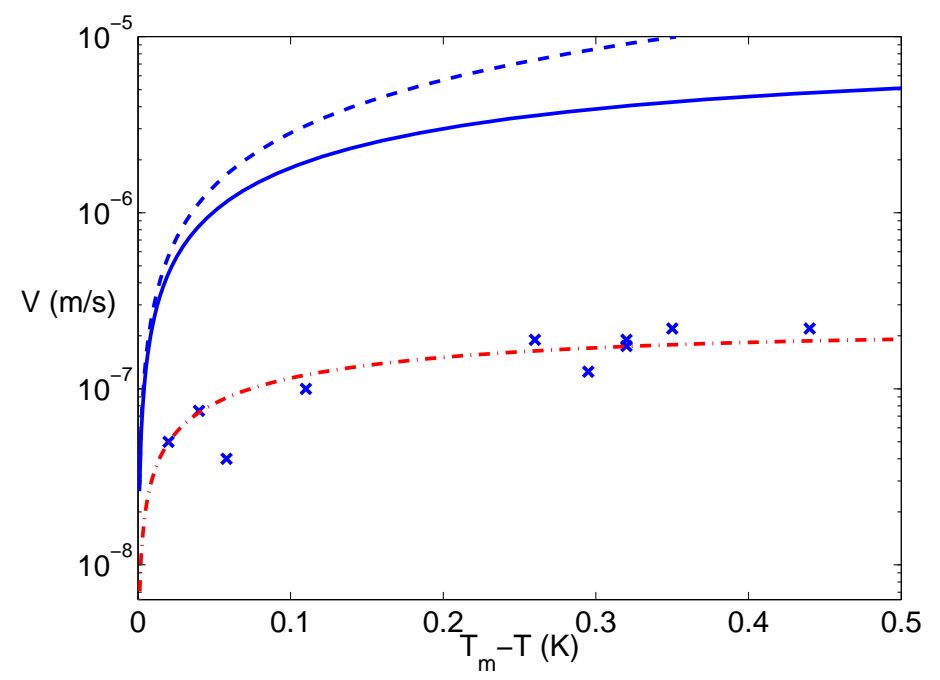

FIGURE 4. Plot showing calculated ice-growth rates in a soil consisting of spherical silica particles. The dashed curve shows the growth rate in the absence of interfacial effects $(\beta=0)$. The continuous curve shows the predicted growth rate using bulk liquid parameters for the premelted films. The dash-dotted curve shows the predicted ice-growth rate with fitted viscosity for the premelted film. Crosses are experimental data from Watanabe (2002).

it is in the bulk liquid (this increase certainly being within the bounds suggested by experiments, such as those performed by Butt et al. (2000) and Goertz et al. (2007)). With this fitting, it can be seen that the predicted ice-growth rates show good agreement with the experimental data. We note that we could also match the data by fitting the thickness of the premelted films while keeping the viscosity equal to that of bulk water. In that case, we would require the premelted film thicknesses to be reduced by a factor of 0.29. Either explanation seems plausible.

From figure 4, we see that there is a significant reduction in the predicted ice-growth rates in the system when the effect of the interfacial dynamics is included, even allowing bulk parameters for the liquid in the premelted films. This means that, as with the soils in figure 3, the interfacial dynamics play an important role in controlling the ice-growth rate. The only exception to this is at very small undercoolings $\left(T_{m}-T \ll 0.01 \mathrm{~K}\right)$. This is because as the ice approaches $T_{m}$, the premelted films thicken, reducing the interfacial resistance to flow and so $\beta \rightarrow 0$.

\subsection{Maximum heave rate in soils}

Figure 4 also allows us to estimate the maximum ice-growth rate in the system. For soils with no frozen fringe, assuming that the growth rate drops off when ice enters the pores between the silica particles, the maximum growth rate $V_{\max }$ can be defined as the highest value of $V(T)$ that occurs while the temperature is greater than the ice-entry temperature (1.2). For Watanabe's particles $T_{m}-T_{i e} \approx 0.45 \mathrm{~K}$ (where this estimate is made following Penner 1973), and so from equation (3.4) the maximum growth rate is $2 \times 10^{-7} \mathrm{~m} / \mathrm{s}$, in agreement with Watanabe's observed value of $2.4 \times 10^{-7} \mathrm{~m} / \mathrm{s}$ (Watanabe 2002). In a soil, the maximum ice-growth rate is important as it is also the maximum heave rate of the soil (e.g. Beskow 1935). This controls how a particular soil responds to freezing and hence its appropriateness for use in engineering applications in cold climates. Frost-susceptible soils have high maximum heave rates, while less susceptible 


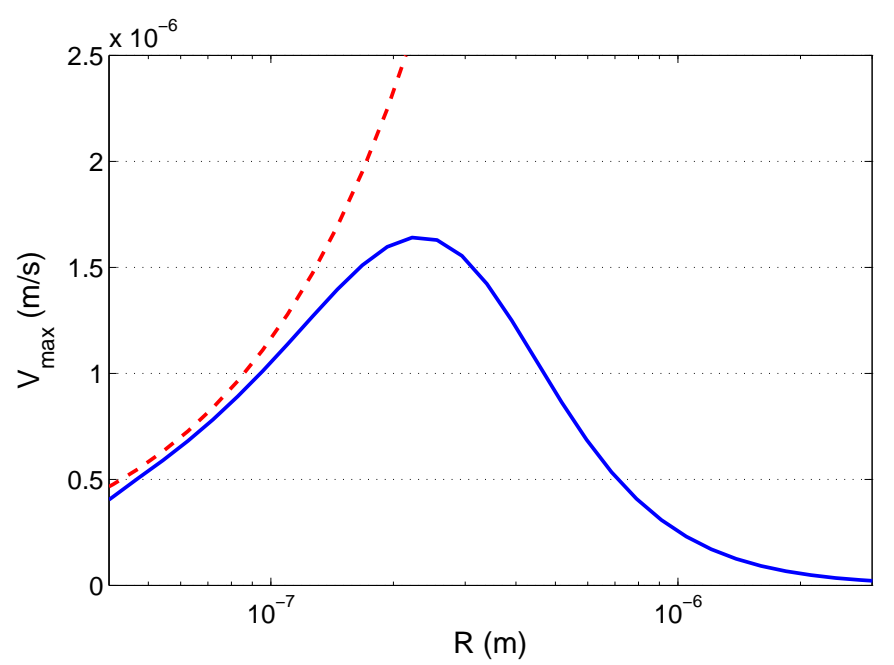

FIgURE 5. The maximum heave rate in a soil consisting of silica particles as a function of the particle size. The dashed curve shows the maximum heave rate when the interfacial resistance are not included $(\beta=0)$, while the continuous curve shows the maximum heave rate including interfacial resistance.

soils have lower heave rates. The maximum heave rate is therefore an extremely useful factor to be able to predict and understand. A particular experimental observation is that frost susceptibility appears to be linked to particle size in the soil. Very fine-grained soils have low permeability which hinders ice growth. In coarse-grained soils, ice enters the soil pores at warm temperatures, blocking them off and reducing flow towards a growing ice lens. Only in medium-grained soils (such as silts) do we find particular high frost susceptibility (e.g. Penner 1968). In order to explain this observation we estimate how $V_{\max }$ would change if we altered the particle size $R$ in Watanabe's experimental system of silica spheres (Watanabe 2002). From the Kozeny-Carman equation (3.6), the permeability is a quadratic function of $R$ so $k=k_{o} R^{2} / R_{o}^{2}$ where $R_{o}$ and $k_{o}$ are the values measured in Watanabe's experiments. From equation (1.2), we have that $T_{m}-T_{i e} \propto 1 / R_{p}$ where $R_{p}$ is the pore radius in between particles. For spherical particles, $R \propto R_{p}$ and so we take $T_{m}-T_{i e}=0.45 R_{o} / R$. We can then calculate the maximum heave rate from equation (3.4) as the largest value of $V(T)$ for $T_{i e}(R) \leqslant T \leqslant T_{m}$. The results are shown in figure 5 for typical parameters taken from table 1 . In particular we have used the fitted value of $\mu_{p}=41 \mu$ from the previous section, and Engemanns data for $d$ given by equation (3.7). Interestingly when we plot the maximum heave rate using the hydrodynamic model without including the interfacial resistance term (dashed curve), we see that the heave rate increases linearly with $R$, leading to unrealistically large heave rates at large $R$. For example $V_{\max }=1.2 \times 10^{-4} \mathrm{~m} / \mathrm{s}$ when $R=10^{-5} \mathrm{~m}$, an extremely unlikely level of heave (e.g. Bell 2000). However when we include the interfacial resistance (continuous curve), the maximum heave rate drops off for large particles, as it is known to do experimentally (Penner 1968). The reason for this can be seen from equation (3.4): for larger particles the interfacial resistance is much higher, and so flow to a growing ice lens is greatly reduced.

To our knowledge, there are currently no experiments that give the maximum heave rate as a function of particle diameter for silica spheres. However this theory provides a straightforward explanation for why the heave rate does not diverge as particles becomes larger. We hope that as data becomes available for premelted film thicknesses on clay 
particles, the theory will also be able to provide quantitative matching with experiments data, such as those commonly used in determining the frost susceptibility of soils by engineers (e.g. Bell 2000).

It should be noted that although our calculation of $V_{\max }(R)$ is for plate-like particles, this behaviour is expected to hold for general particles shapes. By conducting a dimensional analysis on the equations of lubrication theory, we find that $f \sim \mu \tilde{l}^{2} / \tilde{d}^{3}$, where $\tilde{l}$ is the horizontal lengthscale of the film, and $\tilde{d}$ is a typical film thickness. Typically $\tilde{l}=R$, and so the kinetic term $f\left(T_{m}-T\right) \sim R^{2}$. As before $k \sim R^{2}$, and $T_{m}-T_{i e} \sim 1 / R$, and so from equation (2.5) we expect that $V_{\max } \sim R$ for small $R$, while $V_{\max } \sim R^{-3}$ for large $R$, regardless of particle shape. Thus, as with the case of plate-like particles, $V_{\max }$ is expected to drop off when $R$ is small or large. Therefore there is again expected to be a maximum heave rate for intermediate particles where $R$ is not too small that the permeability of the soil cuts off flow to the lens, while $R$ is not too large that the kinetic effect cuts off the flow.

\subsection{Impurities}

As a final note, we emphasise that we have only considered here the kinetic effect in a pure ice-water system. In general cases, there will often be substantial amounts of impurities, such as salts, dissolved in freezing colloidal systems. In this case the film thickness $d$ can have a strong dependence upon the impurity content (e.g. Wettlaufer 1999), and so the kinetic proportionality function $f$ will depend on both temperature and impurity concentration $c$. However this can still be calculated theoretically, or measured experimentally, and then applied to equation (2.5) to determine the expected ice-growth rates $V(c, T)$.

\section{Conclusions}

We have analysed the growth rate of ice-lenses in a freezing porous medium when there is no frozen fringe. As explained in the introduction, in this fringe-less case we expect to see the largest segregated-ice-growth rates (which correspond to the most dangerous heave rates in soils). Often the Clapeyron equation is used to predict the Darcy pressure $p_{f}$ at the ice-lens/soil interface by relating it to the overburden pressure by $p_{f}=P_{o}+\rho L_{m}\left(T_{m}-T\right) / T_{m} \equiv p_{c l}$. In this paper we have demonstrated that this equation is not generally applicable, and that its use will lead to substantial overestimation of heave rates. The key issue that is ignored in making this assumption is the presence of thin, premelted films that separate a growing ice lens from the material that is rejected ahead of it. In order for water to solidify onto the lens, it must flow along these thin films, and we have shown that this adds a considerable contribution to resistance to flow towards a growing lens. This manifests itself as a kinetic-type boundary condition, $p_{c l}-p_{f}=-V f\left(T_{m}-T\right)$, where $f\left(T_{m}-T\right)$, an experimentally-measurable quantity, is a positive function of temperature that depends upon the geometry and constituent material of the porous medium. This shows that $p_{f}$ can only be approximated by the 'equilibrium' value $p_{c l}$ at sufficiently low ice-lens growth rates.

This new, kinetic boundary condition can be used to calculate the ice-growth rate, and this is given by equation (2.5). Interestingly, this equation shows that the ice-growth rate only depends on two quantities, a pressure difference $P_{o}-p_{R}$ that is fixed by external constraints on the system, and the temperature. This is in agreement with experimental observations. Once the form of $f\left(T_{m}-T\right)$ is known, equation (2.5) can be used to predict heave rates at any given pressure and temperature conditions. A very particular example is that the ice-growth rate is zero when $P_{o}=p_{R}+\rho L\left(T_{m}-T\right) / T_{m}$, as is found when 
the kinetic effect is not included. Equation (2.5) shows that the flow of water towards a growing ice lens is resisted by two different factors, firstly the hydraulic resistance to flow of the porous medium, and secondly an interfacial resistance due to flow in the nanoscopic premelted films. The larger of the two resistances controls the flow of water to the ice lens and thus the ice-growth rate.

We have used this theory to calculate the ice-growth rates for a particulate soil consisting of identical, plate-like particles. We have calculated an explicit expression for $f\left(T_{m}-T\right)$ and used it to show that the interfacial resistance typically dominates the hydraulic resistance in common soils. This means that the heave rate in soils is expected to be controlled by the dynamics of flow in the region of the ice-lens/soil interface and not the permeability of the soil itself. Thus calculated ice-growth rates are significantly smaller than those that arise in previous models, in agreement with experiments. We have also used this result for comparison with the experiments of Watanabe (2002) on ice-lens growth in a soil consisting of microscopic silica particles. By using a fitting parameter for the water properties in the premelted films, we see good agreement between theory and experiments. The fitted properties of the water are in line with experimentally observed measurements.

Finally we have used our theory to show how the maximum heave rate depends on the particle size $R$ in a particulate soil. For small $R$ the permeability of the soil is very small, and this limits the flow of water towards a growing ice lens, so that the heave rate is small. At large $R$, the interfacial resistance becomes very large $\left(f\left(T_{m}-T\right) \propto R^{2}\right)$, and this similarly causes the heave rate to fall. This leads to a maximum in the heave rate at intermediate values of $R$. Thus materials consisting of particles of this size are predicted to be particularly susceptible to frost heave. Interestingly this is well known to be the case experimentally, and our theory offers a simple explanation for this phenomenon.

As we have mentioned in the introduction, there are many instances where segregated ice grows in freezing porous medium, ranging from the freezing of soils and rocks to cryopreservation and food preparation. In each of these situations it is important to be able to predict ice-growth rates in order to determine the final structure of the frozen material. In this paper we have demonstrated the (often-overlooked) importance of the interfacial resistance in governing ice growth, in particular showing that it has a significant impact upon ice-growth rates. Thus we hope that this work will be of use in many varied scientific areas in improving understanding of the freezing process.

This publication was based on work supported by Award No KUK-C1-013-04, made by King Abdullah University of Science and Technology (KAUST).

\section{REFERENCES}

Batchelor, G. K. 1967 An Introduction to Fluid Dynamics. Cambridge University Press.

BELL, F. G. 2000 Engineering properties of soils and rocks. Blackwell Science.

Benson, E. E. 2008 Plant cryopreservation: a practical guide, chap. Cryopreservation theory, p. 15. Springer.

Beskow, G. 1935 Soil freezing and frost heaving with special attention to roads and railroads, the swedish geological society, c, no. 375, year book no. 3. technological institute, northwestern university. In Reprinted in Historical Perspectives in Frost Heave Research, P. B. Black and M. J. Hardenberg, eds.,, pp. 37-157. CRREL Special Report 91-23, 1991.

Biermans, M. B. G. M., Dijkema, K. M. \& DE VRies, D. A. 1978 Water movement in porous media towards an ice front. J. Hydrol. 37, 137-148.

Butt, H. J., Doppenschmidt, A., Huttl, G., Muller, E. \& Vinogradova, O. I. 2000 
Analysis of plastic deformation in atomic force microscopy: Application to ice. J. Chem. Phys. 113, 1194.

Dash, J. G., Rempel, A. W. \& Wettlaufer, J. S. 2006 The physics of premelted ice and its geophysical consequences. Rev. Mod. Phys. 78, 695-741.

Deville, S., Saiz, S. \& Tomsia, A. P. 2007 Ice-templated porous alumina structures. Acta Mater. 55, 1965-1974.

Engemann, S., Reichert, H., Dosch, H., Bilgram, J., Honkimaki, V. \& Snigirev, A. 2004 Interfacial melting of ice in contact with $\mathrm{SiO}_{2}$. Phys. Rev. Lett. 92 (20), 205701.

Gao, J., Luedtke, W. D. \& Landman, U. 2000 Structures, solvation forces and shear of molecular films in a rough nano-confinement. Tribology Lett. 9, 3-13.

Goertz, M. P., Houston, J. E. \& Zhu, X. Y. 2007 Hydrophilicity and the viscosity of interfacial water. Langmuir 23, 5491-5497.

KonRAD, J. M. 1987 Procedure for determining the segregation potential of freezing soils. Geotech. Testing J. 10, 51-58.

Miller, R. D. 1977 Lens initiation in secondary heaving. In Proceedings of the International Symposium on Frost Action in Soils, University of Lulea, Sweden, Volume 2.

Ozawa, H. \& Kinosita, S. 1989 Segregated ice growth on a microporous filter. J. Colloid Interface Sci. 132, 113-124.

Penner, E. 1968 Particle size as a basis for predicting frost action in soils. Soils and Foundations $\mathbf{8}, 21-29$.

Penner, E. 1973 Frost heaving pressures in particulate materials. In OECD Symposium on frost action on roads., , vol. 1, pp. 379-385.

Penner, E. \& Goodrich, L. E. 1981 Location of segregated ice in frost-susceptible soil. Eng. Geology 18, 231-244.

Peppin, S. S. L., Elliott, J. A. W. \& Worster, M. G. 2006 Solidification of colloidal suspensions. J. Fluid Mech. 554, 147-166.

Perdigon-Aller, A. C., Aston, M. \& Clarke, S. M. 2005 Preferred orientation in filtercakes of kaolinite. J. Colloid Interface Science 290, 155-165.

Pittenger, B., Fain, S. C., Cochran, M. J., Donev, J. M. K., Robertson, B. E., SzuchMACher, A. \& Overney, R. M. 2001 Premelting at ice-solid interfaces studied via velocitydependent indentation with force microscope tips. Phys. Rev. B 63, 134102.

Rempel, A. W., Wettlaufer, J. S. \& Worster, M. G. 2001 Interfacial premelting and the thermomolecular force: Thermodynamic buoyancy. Phys. Rev. Lett. 87 (8), 088501.

Rempel, Alan W., Wettlaufer, J. S. \& Worster, M. Grae 2004 Premelting dynamics in a continuum model of frost heave. J. Fluid Mech. 498, 227-244.

Roos, Y. H. 1995 Phase transitions in foods. Academic.

Roseberg, R. 2005 Why is ice slippery? Physics Today 58, 50-55.

Seto, J. T. C. \& Konrad, J.-M. 1994 Pore pressure measurements during freezing of an overconsolidated clayey silt. Cold Reg. Sci. Tech. 22, 319.

Style, R. W., Peppin, S. S. L., Cocks, A. C. F. \& Wettlaufer, J. S. ???? Ice-lens formation and geometrical supercooling in soils and other colloidal materials. Submitted .

Style, R. W. \& Worster, M. G. 2005 Surface transport in premelted films with application to grain-boundary grooving. Phys. Rev. Lett. 95, 176102.

Vlahou, I. \& Worster, M. G. 2010 Ice growth in a spherical cavity of a porous medium. J. Glaciol. 56 (196), 271-277.

Watanabe, K. 2002 Relationship between growth rate and supercooling in the formation of ice lenses in a glass powder. J. Cryst. Growth 237-239, 2194-2198.

Watanabe, K. \& Mizoguchi, M. 2000 Ice configuration near a growing ice lens in a freezing porous medium consisting of micro glass particles. J. Cryst. Growth 213, 135-140.

Watanabe, K., Muto, Y. \& Mizoguchi, M. 2001 Water and solute distributions near an ice lens in a glass-powder medium saturated with sodium chloride solution under unidirectional freezing. Cryst. Growth Des. 1, 207-211.

Wettlaufer, J. S. 1999 Impurity effects in the premelting of ice. Phys. Rev. Lett 82 (12), $2516-2519$

Wettlaufer, J. S., Worster, M. G., Wilen, L. A. \& Dash, J. G. 1996 A theory of premelting dynamics for all power law forces. Phys. Rev. Lett 76 (19), 3602-3605. 
Worster, M .G. \& Wettlaufer, J. S. 1999 Fluid Dynamics at Interfaces, chap. The fluid mechanics of premelted liquid films, pp. 339-351. CUP. 



\section{RECENT REPORTS}

26/11 Synaptic bistability due to nucleation and evaporation of receptor clusters

Burlakov

Duričković

Goriely

27/11 Particle trapping and banding in rapid solidification

Elliot

Peppin

28/11 Growth of confined cancer spheroids: a combined experimental and mathematical modelling approach

Loessner

Flegg

Byrne

Hall

Moroney

Clements

McElwain

Hutmacher

29/11 Floating carpets and the delamination of elastic sheets

Wagner

Vella

30/11 Numerical Study of Liquid Crystal Elastomers by a Mixed Finite

Luo

Calderer

31/11 The indentation of pressurized elastic shells: From polymeric cap-

Vella sules to yeast cells

Ajdari

Vaziri

Boudaoud

32/11 Wrinkling of pressurized elastic shells

Vella

Ajdari

Vaziri

Boudaoud

33/11 Data assimilation using bayesian filters and B-spline geological

Duan models

Farmer

Hoteit

$\mathrm{Lu}$

Moroz

34/11 Review of nonlinear Kalman, ensemble and particle filtering with

Luo application to the reservoir history matching problem

Hoteit

Duan

Wang

35/11 Modelling a Tethered Mammalian Sperm Cell undergoing Hyperactivation

Curtis

Kirkman-Brown

Connolly

Gaffney

36/11 A simple mathematical model for investigating the effect of cluster

Zygalakis roots on plant nutrient uptake

Roose

37/11 Frequency jumps in the planar vibrations of an elastic beam

Neukirch

Frelat

Goriely

Maurini 
41/11 On the modelling and simulation of a high pressure shift freezing Smith process

Peppin

Ángel M. Ramos

42/11 An efficient implementation of an implicit FEM scheme for

Burrage fractional-in-space reaction-diffusion equations

Hale

Kay

43/11 Coupling fluid and solute dynamics within the ocular surface tear film: a modelling study of black Line osmolarity

Zubkov

Breward

Gaffney

44/11 A prototypical model for tensional wrinkling in thin sheets

Davidovitch

Schroll

Vella

Adda-Bedia

Cerda

45/11 A fibrocontractive mechanochemical model of dermal wound closure incorporating realistic growth factor

Murphy

Hall

Maini

McCue

McElwain

46/11 A two-compartment mechanochemical model of the roles of transforming growth factor $\beta$ and tissue tension in dermal wound healing

Murphy

Hall

Maini

McCue

McElwain

47/11 Effects of demographic noise on the synchronization of a metapop-

Lai ulation in a fluctuating environment

Newby

Bressloff

48/11 High order weak methods for stochastic differential equations based on modified equations

Abdulle

Cohen

Vilmart

Zygalakis

Copies of these, and any other OCCAM reports can be obtained from:

Oxford Centre for Collaborative Applied Mathematics

Mathematical Institute

24 - 29 St Giles'

Oxford

OX1 3LB

England

www.maths.ox.ac.uk/occam 